\title{
Participación de los equipos de respuesta rápida de tromboembolia pulmonar durante el perioperatorio
}

\author{
Participation of pulmonary embolism response teams during the perioperative period \\ Mateo Porres-Aguilar1*, Javier E. Anaya-Ayala², Flavio A. Grimaldo-Gómez³, Luis E. Santos-Martínez", \\ David Jiménez ${ }^{5}$, Mateo Porres-Muñoz ${ }^{6}$, Raúl Izaguirre-Ávila ${ }^{3}$ y Raúl Carrillo-Esper
}

${ }^{1}$ Division of Adult Thrombosis Medicine, Jewish General Hospital, Department of Medicine, McGill University, Montreal, Quebec, Canadá y Sociedad Mexicana de Trombosis y Hemostasia (SOMETH); ${ }^{2}$ Servicio de Angiología y Cirugía Vascular, Instituto Nacional de Ciencias Médicas y Nutrición Salvador Zubirán; ${ }^{3}$ Departamento de Hematología, Instituto Nacional de Cardiología Ignacio Chávez, Sociedad Mexicana de Trombosis y Hemostasia (SOMETH); ${ }^{4}$ Departamento de Hipertensión Pulmonar y Ventrículo Derecho, Hospital de Cardiología del CMN Siglo XXI, Instituto Mexicano del Seguro Social (IMSS), Ciudad de México, México; ${ }^{5}$ Departamento de Medicina Respiratoria, Hospital Ramón y Cajal y Universidad de Alcalá, Madrid, España; ${ }^{6}$ Beneficencia Española de Tampico, Departamento de Medicina Interna y Geriatría, Tampico; ${ }^{7}$ División de Áreas Críticas, Instituto Nacional de Rehabilitación Guillermo Ibarra, Sociedad Mexicana de Trombosis y Hemostasia (SOMETH), Ciudad de México, México

\begin{abstract}
Resumen
La tromboembolia pulmonar aguda representa una causa frecuente de morbimortalidad cardiovascular, sólo rebasada por los síndromes coronarios agudos y la enfermedad cerebrovascular. El inicio y la intervención de un equipo multidisciplinario de respuesta rápida en la tromboembolia pulmonar son imperantes para mejorar el pronóstico y reducir al mínimo las posibles secuelas en el subgrupo de pacientes más graves. En este artículo de revisión se describe y revisa de manera general el papel actual y potencial que tienen dichos equipos de respuesta rápida, con un enfoque particular en el perioperatorio.
\end{abstract}

Palabras clave: Tromboembolia pulmonar. Equipos de respuesta rápida. Evaluación de resultados. México.

\begin{abstract}
Acute pulmonary embolism represents a frequent cause of cardiovascular morbidity and mortality, only exceeded by acute coronary syndromes and cerebrovascular disease. The start-up and implementation of a designated pulmonary embolism response team is necessary to improve prognosis and minimize long-term sequelae in the subgroup of patients with significant pulmonary embolism. Herein, we describe and discuss an overview of the current and potential role of pulmonary embolism response teams, with a focus on the perioperative period.
\end{abstract}

Key words: Pulmonary embolism. Rapid response teams. Outcomes assessment. Mexico.

Correspondencia:

*Mateo Porres-Aguilar

E-mail: mateo.porres@mail.mcgill.ca
Fecha de recepción: 16-01-2020

Fecha de aceptación: 26-03-2020

DOI: 10.24875/ACM.20000008
Disponible en internet: 14-09-2020 Arch Cardiol Mex. 2020;90(3):321-327

www.archivoscardiologia.com 1405-9940/๑ 2020 Instituto Nacional de Cardiología Ignacio Chávez. Publicado por Permanyer. Este es un artículo open access bajo la licencia CC BY-NC-ND (http://creativecommons.org/licenses/by-nc-nd/4.0/). 


\section{Introducción}

La tromboembolia pulmonar (TEP) aguda representa la tercera causa más frecuente de mortalidad cardiovascular en el mundo occidental, después de los síndromes coronarios agudos y la enfermedad cerebrovascular $^{1-5}$. La clasificación y estratificación del riesgo, apropiadas, rápidas y precisas en la TEP aguda, representan sin duda alguna la piedra angular para tomar decisiones clínicas en el abordaje terapéutico y el pronóstico. La mortalidad de la TEP de riesgo intermedio-alto (submasiva) y alto es todavía elevada. La TEP aguda de alto riesgo, conocida antes como TEP masiva, se define en clínica como una franca inestabilidad hemodinámica manifestada por hipotensión persistente (presión arterial sistólica $<90 \mathrm{mmHg}$ o choque cardiogénico) y tiene una mortalidad de 30 a $50 \%{ }^{6}$.

\section{Importancia de la clasificación actual y estratificación del riesgo de la TEP aguda}

En fecha reciente, las guías clínicas de la European Society of Cardiology (ESC) propusieron un importante ajuste dentro de la clasificación de la TEP aguda de riesgo intermedio, al subdividir a los pacientes en riesgo intermedio-alto e intermedio-bajo ${ }^{4}$. El primer subgrupo se caracteriza por disfunción ventricular derecha objetiva y elevación de los marcadores de daño miocárdico (p. ej., elevación de troponinas y péptido natriurético cerebral); el segundo se distingue por tener tan sólo uno de los parámetros ya mencionados o ningún de ellos. La TEP aguda de riesgo intermedio-alto posee una mortalidad de 10 a $25 \% \%^{7,8}$. Existe hoy en día una controversia importante en múltiples sociedades médicas y entre los propios investigadores clínicos acerca de cómo mejorar la definición de TEP aguda de riesgo intermedio-alto, dadas las diferentes definiciones heterogéneas en diversos estudios y protocolos clínicos en la última década. Con base en lo anterior, es imperativo y necesario formular pronto una definición uniforme y homogénea basada en un consenso sustentado por un grupo de expertos mundial en TEP aguda, tal y como existe y se ha validado una definición universal y en constante evolución del infarto agudo de miocardio, con la finalidad común de poder identificar al subgrupo de pacientes con TEP aguda de riesgo intermedio-alto en verdad grave o enfermos con peor pronóstico a corto plazo (p. ej., mortalidad o recurrencia de tromboembolia venoso a 30 días) o una evolución clínica intrahospitalaria tórpida con deterioro clínico progresivo a pesar de iniciar la hospitalización en estado "normotenso" con la institución de anticoagulación temprana ( $<12$ horas tras ingresar a urgencias).

Es de suma importancia la propuesta de llevar a cabo un abordaje multimodal adecuado, rápido y preciso en la gradación del riesgo y el pronóstico, que incluya criterios clínicos, intuición del clínico en la sospecha del deterioro clínico rápido y progresivo, sustentado y apoyado con escalas clínicas de gravedad de la TEP aguda, demostración objetiva de disfunción ventricular derecha por ecocardiografía o tomografía computarizada torácica contrastada y elevación de biomarcadores de daño miocárdico ${ }^{9}$. Los equipos de respuesta rápida en tromboembolia pulmonar (ERRTEP) tienen la capacidad de completar, analizar e integrar dicha estratificación del riesgo combinada y su finalidad es poder detectar de manera temprana a los pacientes más críticos y los que tienen en realidad una mortalidad elevada a corto plazo en el período intrahospitalario, lo que supone un peor pronóstico; de esta forma puede idearse un plan de abordaje terapéutico personalizado/individualizado de acuerdo con las características particulares de cada paciente, así como la detección de pacientes en quienes es necesaria una modalidad terapéutica más radical, por ejemplo la necesidad de procedimientos de reperfusión temprana ${ }^{10}$.

\section{Razones fundamentales para la creación, organización y desempeño de los ERRTEP}

El Massachusetts General Hospital (MGH) de Boston, Massachusetts, introdujo los ERRTEP en 2012 11-15. Con posterioridad, otros centros de tercer nivel tanto académicos como comunitarios en EE.UU. empezaron a formar, organizar, estructurar e incorporar sus respectivos ERRTEP. En mayo del 2015 se fundó la institución nacional PERT (Pulmonary Embolism Response Teams) en EE.UU., con los objetivos primordiales de promover de manera continua el modelo de los ERRTEP, guiar a líderes de cada equipo a practicar con excelencia el cuidado clínico, el abordaje diagnóstico y terapéutico, y el pronóstico, generar una base de datos sólida para la investigación clínica y los estudios multicéntricos que fortalecieran la medicina basada en evidencias en cuanto a la actualización en el cuidado de la TEP aguda, así como crear y aplicar programas de mejora de la calidad para pacientes con TEP aguda grave y compleja ${ }^{16,17}$. En la actualidad existen ya más de 140 instituciones académicas y hospitalarias en EE.UU y en el mundo entero afiliadas y acreditadas por la agrupación PERT ${ }^{16,17}$. 


\section{Estructura y funcionamiento de los ERRTEP}

Por lo general, los ERRTEP están conformados por un equipo multidisciplinario de diversas especialidades, con un interés particular común en tromboembolismo venoso, e incluyen sobre todo medicina vascular y trombosis de adultos, medicina de urgencias, medicina interna, cardiología invasiva y radiología vascular diagnóstica e intervencionista, neumología/terapia intensiva, así como cirugía cardiotorácica y vascular. Hay que enfatizar que no debe contarse en todos los casos con la totalidad de las especialidades mencionadas; en realidad, según sean los recursos de cada institución hospitalaria, el número de integrantes de un ERRTEP puede ser desde dos a tres expertos hasta uno más extenso de ocho a 10. A continuación se esquematizan de forma nemotécnica los principales integrantes de un ERRTEP por sus siglas en inglés: Pulmonary Embolism Response Team (PERT):

$-\mathrm{P}=$ Pulmonologist/intensivist (neumología y terapia intensiva)

- $\mathrm{E}=$ Endovascular expert (cardiología o radiología intervencionista)

- $\mathrm{R}=$ Diagnostic radiology (radiología diagnóstica)

$-\mathrm{T}=$ Thrombosis expert (medicina vascular, trombosis de adultos o hematología)

Uno de los objetivos principales de los ERRTEP consiste en facilitar de manera rápida, efectiva, precisa, eficaz y consensuada entre los miembros del equipo la formulación/emisión de un plan terapéutico y atención óptima, razonable y personalizada para un paciente en particular con TEP aguda grave compleja (riesgos intermedio-alto y alto), posterior a la evaluación y análisis del riesgo respecto del beneficio dentro de las alternativas y modalidades terapéuticas existentes, sobre todo cuando se considere la posible utilización de medidas de reperfusión temprana en pacientes con choque cardiogénico o con posibilidad de deterioro rápido progresivo en las siguientes 24 a 48 horas $^{18}$.

Cualquier médico 0 residente en formación puede activar el código y la consulta para los ERRTEP dentro o fuera del hospital; el residente o el médico especialista que se encuentren de guardia exclusivamente para los consultas ligadas a los ERRTEP deben evaluar, analizar e integrar dicha consulta en un lapso no mayor de 90 minutos; a continuación se presenta y revisa el caso entre los miembros del equipo, se establece una conclusión y se toman decisiones clínicas en cuanto al tratamiento y el pronóstico por consenso entre los miembros del equipo, y se comunica inmediatamente al médico consultante en tiempo real y de manera directa.
La tabla 1 enlista las principales ventajas (pros) y obstáculos (cons) posibles dentro del funcionamiento y desempeño de los ERRTEP.

\section{Resultados iniciales de los ERRTEP}

Dados los avances significativos en la ciencia, el conocimiento y la tecnología sobre las medidas terapéuticas de reperfusión en la TEP aguda, como los trombolíticos sistémicos más los específicos de fibrina, dispositivos de trombólisis dirigida por catéter regular o asistida por ultrasonido, la trombectomía percutánea por aspiración o mecánica asistida por catéter, la embolectomía pulmonar quirúrgica abierta y el apoyo hemodinámico avanzado, se requiere una comunicación multidisciplinaria entre diversas especialidades médicas y quirúrgicas basadas en el trabajo en equipo, la cual ha crecido de forma notoria ${ }^{11-15}$.

A partir de lo anterior, y junto al advenimiento, rápido crecimiento, desarrollo y constante evolución de los ERRTEP, a continuación se describen algunos de los primeros estudios que mejor caracterizan y describen el desempeño de los ERRTEP.

Kabrhel, et al. ${ }^{13}$ describieron de manera retrospectiva el funcionamiento de los ERRTEP del MGH de Boston en 394 pacientes en los cuales se activaron los ERRTEP; en 217 pacientes (69\%) se recomendó la anticoagulación, en $28(9 \%)$ la trombólisis asistida por catéter, en $15(8 \%)$ la trombólisis sistémica y en $8(3 \%)$ la embolectomía pulmonar quirúrgica. Cincuenta y tres por ciento de las consultas o activaciones de los ERRTEP tuvo lugar durante la noche y los fines de semana; la aceptación y adopción de los ERRTEP se incrementaron de manera sostenida, con un aumento del número de activaciones en un $16 \%$ cada seis meses. La mortalidad por cualquier causa fue del $12 \%$ en los pacientes con demostración objetiva de TEP aguda.

El grupo de la Universidad de Nueva York publicó en 2017 su experiencia con sus 124 activaciones de ERRTEP en 20 meses $^{19}$; el $90.8 \%$ de sus pacientes pertenecía al subgrupo de TEP aguda de riesgo intermedio-alto y el $9.2 \%$ a la TEP aguda de alto riesgo. Dentro de los tratamientos de reperfusión se utilizaron la trombólisis asistida por catéter en 25 pacientes (20\%), trombólisis sistémica en 6 (5\%) y anticoagulación sólo en 54 (44\%). Se registró una mortalidad intrahospitalaria del $13 \%$, con cinco episodios hemorrágicos mayores: un paciente que recibió atención asistida por catéter, un paciente sometido a trombólisis sistémica y tres sólo bajo anticoagulación.

En fecha reciente, Rosovsky, et al..$^{20}$ publicaron un análisis retrospectivo de 10 años, el cual evaluó los 
Tabla 1. Ventajas y obstáculos durante la creación, organización y desempeño de los equipos de respuesta rápida en tromboembolia pulmonar (ERRTEP)

\section{Ventajas (PROS)}

- Los ERRTEP generan un plan muy valioso con respecto a las recomendaciones diagnósticas y terapéuticas más adecuadas para los casos complejos de TEP aguda grave

- Reúnen a un equipo multidisciplinario de expertos, el cual revisa y analiza de manera crítica el riesgo/beneficio en relación con las diversas alternativas terapéuticas y de atención, hasta alcanzar un consenso en la gran mayoría de los casos consultados

- Facilita de manera efectiva la movilización de recursos diagnósticos y terapéuticos dentro de una institución hospitalaria

- Participa de manera proactiva en el tratamiento intrahospitalario de pacientes con TEP aguda grave, incluida la selección adecuada en el régimen de anticoagulación a largo plazo, y coordina el seguimiento apropiado en la consulta externa

- Sustenta y apoya las fortalezas de cada subespecialidad que interviene en dichos equipos para promover la cohesión de los ERRTEP

- Genera una gran base de datos clínicos y epidemiológicos, en favor de la investigación clínica y mejora continua de la calidad intrahospitalaria en el cuidado de pacientes con tromboembolismo venoso

- Identifica a grupos de interés particular en tromboembolismo venoso dentro de las subespecialidades existentes en instituciones hospitalarias educativa/académicas

- Acepta y adopta de modo global/mundial los ERRTEP en hospitales e instituciones académicas y privadas

- Favorece el posible cambio en el paradigma del abordaje diagnóstico y terapéutico en la TEP aguda, sobre todo en sus formas más graves Obstáculos/retos (CONS)

- Falta de interés y compromiso, lo que resulta en una falla importante durante la construcción e inicio o desempeño de un ERRTEP

- No alcanza un consenso o decisión final entre los especialistas que conforman un ERRTEP; la consecuencia es el retraso significativo en el abordaje terapéutico más adecuado, sobre todos los pacientes que se encuentran en estado crítico

- Barreras u obstáculos durante la difusión y expansión del concepto ERRTEP, sea intramuros (dentro del hospital o institución académica en donde se inicia dicho equipo) o extramuros (en otros hospitales locales o regionales)

- Percepción falsa de que los ERRTEP se crearon sólo para el uso desmedido o abuso de procedimientos endovasculares dentro del tratamiento de la TEP aguda grave

- Falta de colaboración, interés o compromiso por parte de los residentes en formación (p. ej., personal de urgencias, internistas, neumólogos, cardiólogos o hematólogos) como potenciales líderes y participación proactiva en un ERRTEP

- Aspectos por resolver, como la compensación económica y la remuneración adecuadas al formular un plan de abordaje diagnóstico y terapéutico apropiado por parte de un ERRTEP

patrones terapéuticos y pronósticos en los pacientes con TEP aguda grave (riesgo intermedio-alto y alto), al comparar la era anterior a ERRTEP (2006-2012) con la posterior a ERRTEP (2012-2016); más pacientes se sometieron a una conducta terapéutica de reperfusión radical temprana ( $1 \%$ vs. $14 \% ; p=<0.0001$ ) durante la era posterior a ERRTEP; sin embargo, no hubo una diferencia significativa en cuanto a los episodios de hemorragia mayor o letal, así como en cuanto a la diferencia en la mortalidad por cualquier causa.

También en fecha reciente se publicó el primer análisis retrospectivo multicéntrico de los ERRTEP en EE.UU., el cual incluyó a 418 pacientes documentados del registro activo de la organización estadounidense PERT de octubre de 2016 a octubre de 2017; el número de activaciones mensuales que incluyó a los ERRTEP fueron de 3 a 13 consultas mensuales; la mayoría de las activaciones ocurrió en pacientes con TEP de riesgo intermedio-bajo e intermedio-alto, con $34 \%$ y $35 \%$, respectivamente; y $12 \%$ de dichas activaciones se realizó en pacientes con TEP de alto riesgo/masiva. Existió una variabilidad considerable en cuanto a la utilización de medidas de reperfusión entre diferentes ERRTEP (16 al $44 \%$ ), con una mortalidad a 30 días del $16 \%{ }^{21}$.

La figura 1 es un diagrama de flujo que esquematiza de manera integral el funcionamiento de los ERRTEP.

\section{Papel potencial de los ERRTEP en el perioperatorio}

Dada la introducción reciente de los ERRTEP en la práctica diaria clínica, todavía no existe evidencia 


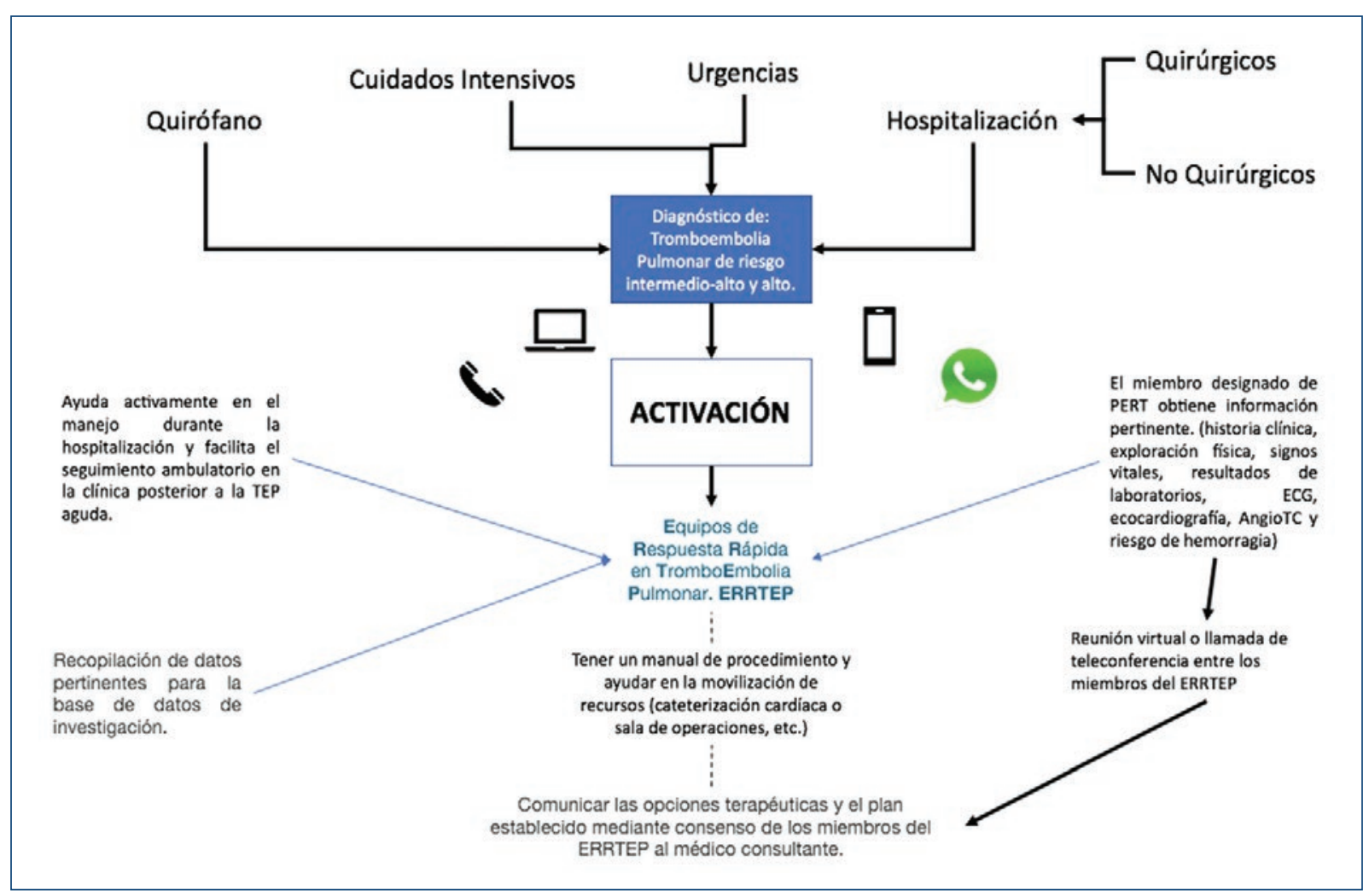

Figura 1. Diagrama de flujo que esquematiza de manera integral el funcionamiento de los ERRTEP.

sólida sustentada en las publicaciones médicas que analizan el potencial papel de los ERRTEP en el tratamiento de pacientes complejos en el perioperatorio.

Es importante disponer de un trabajo colaborativo y coordinado de los ERRTEP con los servicios específicos designados en medicina de trombosis; estos últimos se han desarrollado en algunos países como Canadá. Quizás podría considerarse como perspectiva futura que los ERRTEP colaboren en el tratamiento perioperatorio farmacológico de los anticoagulantes orales directos como antagonistas de la vitamina $\mathrm{K}$, ya que la atención perioperatoria de dichos anticoagulantes puede resultar muy compleja y confusa para la mayoría de los médicos. Son interrogantes importantes las siguientes: ¿es necesario suspender el anticoagulante oral para algún procedimiento u operación?; si la respuesta es afirmativa, ¿cuándo es conveniente hacerlo?, ¿se requiere de manera perioperatoria ajuste, como en el puenteo (bridging) en el régimen de anticoagulación en pacientes que consumen antagonistas de la vitamina $K$ ?, ¿cuándo es conveniente reiniciar el anticoagulante en el postoperatorio? ${ }^{22,23}$ Estas preguntas podrían abordarse en los ERRTEP, en estrecha interrelación también con los químicos farmacéuticos con experiencia en antitrombóticos, los cuales también deben considerarse como miembros proactivos de los ERRTEP.

Los ERRTEP podrían tener una participación en la evaluación perioperatoria del riesgo tromboembólico y hemorrágico en pacientes médicos y quirúrgicos, ya sea en sujetos previamente anticoagulados 0 individuos complejos en quienes se requiera una evaluación enfocada en la tromboprofilaxis apropiada intrahospitalaria, así como la necesidad de la extensión tromboprofiláctica farmacológica más allá del alta hospitalaria (p. ej., tromboprofilaxis farmacológica extendida de 30 a 45 días), si ésta estuviera indicada con base en escalas de riesgo tromboembólico.

Otra de las indiscutibles y valiosas actividades en las cuales los ERRTEP pueden ser piedra angular es la relacionada con la estratificación del riesgo, rápida, correcta y eficiente, además de la detección de factores de riesgo significativos que modifiquen la morbimortalidad en los pacientes diagnosticados con TEP aguda grave perioperatoria. El grupo de estudio de la Sociedad Japonesa de Anestesiología describió en fecha reciente, en un análisis retrospectivo de 214 pacientes, los factores de riesgo perioperatorios 
Tabla 2. Potenciales funciones de los equipos de respuesta rápida en tromboembolia pulmonar (ERRTEP) durante el perioperatorio

\footnotetext{
- Evaluación perioperatoria del riesgo tromboembólico, identificación de pacientes con riesgo intermedio y alto para dichas complicaciones, así como extensión de recomendaciones de tromboprofilaxis apropiadas

- Recomendaciones en el tratamiento perioperatorio de anticoagulantes orales directos, así como de acenocumarínicos en enfermos previamente anticoagulados

- Identificación de pacientes que requieren de manera perioperatoria ajuste por un riesgo tromboembólico elevado, incluido el puenteo en su régimen de anticoagulación

- Determinación del riesgo tromboembólico y hemorrágico en pacientes médicos y quirúrgicos considerados para iniciar tratamiento con anticoagulantes

- Participación en la estratificación del riesgo rápida y adecuada, el abordaje terapéutico y el pronóstico apropiado en pacientes detectados con TEP aguda grave perioperatoria

- Evaluación de los beneficios respecto de los riesgos de pacientes considerados para someterse a trombólisis sistémica o guiada por catéter, enfocada en factores de riesgo específicos que predigan el desarrollo de hemorragia grave mayor o intracraneal
}

significativos vinculados con una mortalidad a 30 días elevada, entre ellos los siguientes: sexo masculino, edad mayor avanzada > 80 años, inmovilización prolongada, antecedente de insuficiencia cardíaca, así como el nulo consumo previo de anticoagulantes ${ }^{24}$. La tabla 2 propone las potenciales funciones de los ERRTEP en el perioperatorio.

El tratamiento de pacientes con TEP aguda grave compleja es motivo frecuente de controversia en virtud de la heterogeneidad de los enfermos, la disponibilidad de modalidades terapéuticas y recursos con los que cada institución hospitalaria cuenta, la ausencia de guías consensuadas y en ocasiones la falta de especialistas en el segundo nivel de atención. Aunque la introducción de ERRTEP ha emergido como un abordaje estandarizado, el efecto hasta ahora no es del todo claro. En un estudio reciente, Chaudhury, et al. condujeron un análisis retrospectivo que comparó los resultados 18 meses antes y después del inicio de ERRTEP; los autores observaron en una cohorte de 769 pacientes una disminución significativa de la mortalidad hospitalaria a 30 días, la cual era más pronunciada en sujetos de riesgo intermedio-alto y alto (antes de ERRTEP de $10.0 \%$ vs. después de ERRTEP de $5.3 \%$; $p=0.02$ ), la cual fue muy notoria en los pacientes sometidos a algún tipo de tratamiento de reperfusión temprana (p. ej., trombólisis sistémica o tratamiento endovascular dirigido por catéter), lo que sugiere que esta población es la más beneficiada de los ERRTEP ${ }^{25}$.

Todavía existen muchos resultados contrastantes entre diversos grupos de ERRTEP; sin embargo, la adecuada utilización, acumulación de experiencia a través de la exposición de casos, así como la consistencia en la excelencia del cuidado de los pacientes con tromboembolismo venoso de los ERRTEP sin duda mostrará en el futuro a mediano y largo plazos sus efectos positivos en la morbimortalidad en la TEP aguda, en particular en grupos de riesgo intermedio-alto y alto.

\section{Conclusiones}

Los ERRTEP se diseñaron con el fin de ofrecer, a partir de una evaluación detallada y multidisciplinaria, el plan óptimo de abordaje diagnóstico, terapéutico y pronóstico en pacientes complejos con TEP aguda grave, de una forma personalizada para cada escenario clínico.

Estos equipos representan no sólo un concepto, sino un proceso único, auténtico e innovador en constante desarrollo y evolución, cuyo objetivo principal es que se adopten no sólo en EE.UU. sino en todo el mundo ${ }^{26}$. A medida que más evidencia científica robusta se acumule, y exista mejor desempeño de dichos equipos, será posible un efecto positivo significativo para abatir la elevada mortalidad en los pacientes con TEP aguda de riesgo intermedio-alto y alto; de ese modo se mejorará la calidad de vida y el pronóstico y se cambiará el paradigma en el cuidado de esta devastadora enfermedad.

\section{Financiamiento}

Ninguno.

\section{Conflicto de intereses}

Los autores declaran no tener ningún conflicto de intereses. 


\section{Responsabilidades éticas}

Protección de personas y animales. Los autores declaran que para esta investigación no se han realizado experimentos en seres humanos ni en animales.

Confidencialidad de los datos. Los autores declaran que en este artículo no aparecen datos de pacientes.

Derecho a la privacidad y consentimiento informado. Los autores declaran que en este artículo no aparecen datos de pacientes.

\section{Bibliografía}

1. Teleb M, Porres-Aguilar M, Anaya-Ayala JE, Rodríguez-Castro C, Porres-Muñoz M, Mukherjee D. Potential role of systemic thrombolysis in acute submassive intermediate risk pulmonary embolism: review and future perspectives. Ther Adv Cardiovasc Dis. 2016;10(2):103-110.

2. Go AS, Mozaffarian D, Roger VL, Benjamin EJ, Berry JD, Blaha MJ, et al. Executive summary: heart disease and stroke statistics-2014 update: a report from the American Heart Association. Circulation. 2014; 129(3):399-410.

3. Cohen AT, Agnelli G, Anderson FA, Arcelus JI, Bergqvist D, Brecht JG, et al. Venous thromboembolism (VTE) in Europe. The number of VTE events and associated morbidity and mortality. Thromb Haemost. 2007:98(4):756-64.

4. Konstantinides SV, Torbicki A, Agnelli GC, Danchin N, Fitzmaurice D, Galie N, et al. 2014 European Society of Cardiology (ESC) guidelines on the diagnosis and management of acute pulmonary embolism. Eur Heart J. 2014;35(43):3033-3069

5. Mahan CE, Borrego ME, Woersching AL, Federici R, Downey R. Venous thromboembolism: annualised United States models for total, hospital-acquired and preventable costs utilising long-term attack rates. Thromb Haemost. 2012;108(2):291-302.

6. Porres-Aguilar M, Anaya-Ayala JE, Heresi GA, Rivera-Lebron BN. Pulmonary embolism response teams: a novel approach for the care of complex patients with pulmonary embolism. Clin Appl Thromb Hemost. 2018;19:1076029618812954. doi: 10.1177/1076029618812954.

7. Jiménez D, Bikdeli B, Marshall PS, Tapson VF. Aggressive treatment of intermediate-risk patients with acute symptomatic pulmonary embolism. Clin Chest Med. 2018;39(3):569-581.

8. Kearon C, Akl EA, Ornelas J, Blaivas A, Jiménez D, Bounameaux $\mathrm{H}$, et al Antithrombotic therapy for VTE disease: CHEST Guideline and Expert Panel Report. Chest. 2016;149(2):315-352.

9. Kosova EC, Desai KR, Schimmel DR. Endovascular management of massive and submassive acute pulmonary embolism: current trends in risk stratification and catheter-directed therapies. Curr Cardiol Rep. 2017; 19(6):54.
10. Porres-Aguilar M, Anaya-Ayala JE, Santos-Martínez LE. Intermediate-high risk pulmonary embolism: redefining it better with the support of the pulmonary embolism response teams approach. Arch Cardiol Mex. 2019;89(1):53-55.

11. Provias T, Dudsinzki DM, Jaff MR, Rosenfield K, Channick RN, Baker J, et al. The Massachusetts General Hospital Pulmonary Embolism Response Team (MGH PERT): creation of a multidisciplinary program to improve care of patients with massive and submassive pulmonary embolism. Hosp Pract. 1995;2014;42(1):31-37.

12. Dudzinski DM, Giri J, Rosenfield K. Interventional therapies for pulmonary embolism. Circ Cardiovasc Interv. 2017;10(2).pii: e004345. doi: 10.1161/ CIRCINTERVENTIONS.116.004345.

13. Kabrhel C, Rosovsky R, Channik RN, Jaff MR, Weinberg I, Sundt T, et al. A multidisciplinary pulmonary embolism response team: initial 30-month experience with a novel approach to delivery of care to patients with submassive and massive pulmonary embolism. Chest. 2016;150(2): 384-393.

14. Witkin AS. Acute and chronic pulmonary embolism: the role of the pulmonary embolism response team. Curr Opin Cardiol. 2017;32(6):672-678.

15. Witkin AS, Harshbarger S, Kabrhel C. Pulmonary embolism response teams. Semin Thromb Hemost. 2016;42(8):857-864. Epub 2016 Oct 21. Review.

16. Available from: https://pertconsortium.org/about/(Accessed on July 15, 2019)

17. Serhal M, Haddadin IS, Heresi GA, Hornacek DA, Shishebhor MH, Bartolomew JR. Pulmoanry embolism response teams. J Thromb Thrombolysis. 2017;44(1):19-29.

18. Teleb M, Porres-Aguilar M, Rivera-Lebron BN, Ngamdu KS, Botrus G, Anaya-Ayala JE, et al. Ultrasound-assisted catheter-directed thrombolysis: a novel and promising endovascular therapeutic modality for intermediate-risk pulmonary embolism. Angiology. 2017;68(6):494-501.

19. Sista AK, Friedman OA, Dou E, Denvir B, Askin B, Stern J, et al. A pulmonary embolism response team's initial 20 -month experience treating 87 patients with submassive and massive pulmonary embolism. Vasc Med. 2018;23(1):65-71.

20. Rosovsky R, Zhao K, Sista A, Rivera-Lebron B, Kabrhel C. Pulmonary embolism response teams: purpose, evidence for efficacy, and future research directions. Res Pract Thromb Haemost. 2019;3(3):315-330.

21. Schultz J, Giordano N, Zheng H, Parry BA, Barnes GD, Heresi GA, et al. EXPRESS: a multidisciplinary pulmonary embolism response team (PERT) - Experience from a national multicenter consortium. Pulm Circ. 2019:2045894018824563. doi: 10.1177/2045894018824563. [Epub ahead of print

22. Leitch J, van Vlimen J. Managing the perioperative patient on direct oral anticoagulation. Can J Anesth. 2017;64:656-672.

23. Barnes GD, Mouland E. Peri-procedural management of oral anticoagulants in the DOACs era. Prog Cardiovasc Dis. 2018;60:600-606.

24. Working group for audit about perioperative accident and pulmonary embolism of Japanese Society of Anesthesiologists. Perioperative risk factors for death among patients with symptomatic pulmonary thromboembolism. J Anesth. 2017;31(3):478-482.

25. Chaudhury P, Gadre SK, Schneider E, Renapurkar RD, Gomes M, Haddadin I, et al. Impact of multidisciplinary pulmonary embolism response team availability on management and outcomes. Am J Cardiol. 2019;124(9):1465-1469.

26. Porres-Aguilar M, Rivera-Lebron B, Anaya-Ayala JE, Guerrero de León MC, Mukherjee D. Perioperative acute pulmonary embolism: a concise review with emphasis on multidisciplinary approach. Int J Angiol. 2020;29: (In Press; pending DOI). 